\title{
Correlation between electronic structure, mechanical properties and phase stability in intermetallic compounds
}

\author{
P RAVINDRAN and R ASOKAMANI* \\ Department of Physics, Anna University, Madras 600025 , India
}

\begin{abstract}
From the detailed electronic structure studies on intermetallic compounds, it has been found that these materials have low heat of formation and large glass-forming ability, if the Fermi level falls on the peak in the density of states (DOS) curve. On the other hand, if the $E_{F}$ falls on the pseudogap in the DOS curve, the ordering energy will be larger and the system prefers to form an ordered alloy. The first principles electronic structure calculations performed on $\mathrm{Ni}_{3} \mathrm{Al}$ show that it is possible to vary the filling of bonding, nonbonding and antibonding states in the DOS curve and this in turn shows gradual structural transformation as well as formation of multiphases by ternary alloying. Possibilities of tailoring the properties of materials by tuning the Fermi level is discussed in this paper.
\end{abstract}

Keywords. Phase stability; pseudogap; electronic structure; density of states; Fermi level.

\section{Introduction}

A giant leap in bridging the gap between the macroscopic mechanical properties and microscopic quantum physics was made only in the mid-seventies by Moruzzi et al (1978). They made use of the Koringa-Kohn-Rostoker theory to obtain the electronic structure of different solids and to calculate their cohesive energies and bulk moduli. Very soon linear methods such as the linear muffin tin orbital (LMTO) and linear augmented plane wave (LAPW) methods overtook them because of their computational simplicity (Andersen 1975). The most important milestone in this area was made by Skriver, who using the LMTO method made a systematic study of the relative stability of the elemental solids as found in the periodic table (Skriver 1985). He could establish the correlation between the structural sequence and the $d$ electron number, which was also pointed out by Pettifor $(1970,1992)$ from his phenomenological approach. The linear methods since have made substantial progress and have been used to predict structural transformation of solids under high pressure. Freeman and his collaborators made extensive studies on various aspects of the ordered intermetallic compounds of technological importance. They explained the relative stability between the structures by comparing their total energies and, in addition, calculated quantities such as antiphase boundary energy (APB), stacking fault (SF) energy, the elastic constants (Hong et al 1991; Xu and Freeman 1991; Xu et al 1991) etc, which lie in the realm of physical metallurgy.

The $\mathrm{Ti}$ and Ni-based intermetallic compounds have desirable properties for use in high temperature structural applications. The biggest hurdle for using these materials in structural applications is their poor ductility. The brittleness of intermetallic compounds mainly arises from intergranular fracture or the intragranular fracture. The first of these two can be due to the grain boundary weakness and the latter is due to the slip incompatibility at the grain boundary arising from the lack of sufficient numbers of

\footnotetext{
*Author for correspondence
} 
operable independent slip systems to satisfy the Von Mises criterion for ductility (Von Mises and Angew 1928). By ternary alloying, one can strengthen the grain boundaries.

The electronic structure studies give valuable information about the formation of alloys, their phase stability and various physical properties quantitatively. At present, attempts are in progress to apply first-principles microscopic theories, such as the local density theory, to the problem of understanding the structural behaviours, phase diagrams, site occupancy of alloying elements, formation of various defects and mechanical properties of structural intermetallics.

Still there is much to be done theoretically to correlate improvements in the mechanical properties with changes in the associated microscopic electronic structure, especially for some of the more important aluminide intermetallic compounds (e.g. $\mathrm{Ni}-\mathrm{Al}, \mathrm{Ti}-\mathrm{Al}, \mathrm{Zr}-\mathrm{Al}$ and $\mathrm{Nb}-\mathrm{Al}$ ). The understanding of their underlying physics which governs strength, ductility and resistance to fracture and thermal failure is far from complete. The aim of our current work has been to correlate the electronic structure of structural intermetallics with their various physical properties. We discuss in this paper some of the microscopic reasons for the formation of ordered or disordered phases of the alloys and highlight the possibilities of tailoring the properties of intermetallics.

\section{The concept of pseudogap and bonding}

In most of the ordered intermetallic compounds a deep valley in the density of states (DOS) curve in the vicinity of Fermi level is observed and this deep valley is called pseudogap. The origin of the pseudogap in ordered intermetallic compounds is traced to (i) charge transfer or ionicity, (ii) hybridization or covalence and (iii) $d$-resonance (Gelatt et al 1983; Pastural et al 1985).

If the electronegativity difference between the constituents is large, due to screening or charge neutrality consideration, a drastic alteration in band shape takes place due to the redistribution of electrons in energy. As a consequence, the screening electrons are assigned mostly to low-lying states in the band, leading to the creation of a minimum in the DOS curve. If the constituents in the intermetallic compounds offer electrons in the same energy range, their wave functions are strongly mixed with each other and this covalent hybridization increases the bond strength, implying a transfer of electrons to lower energy range, thereby producing a pseudogap. In transition metals and their compounds, the presence of narrow $d$-states near the Fermi level pulled towards lower energy range from Fermi level due to resonance effect and, hence, deep valley closer to $E_{\mathrm{F}}$ appears. As most of the intermetallic compounds have mixed bonding nature, the combined effects of the above mechanisms are responsible for the creation of pseudogap.

\section{Correlation between the position of $E_{\mathrm{F}}$ with respect to pseudogap and stability}

Band structure calculations of several intermetallics show that the structural stability of the intermetallic compounds is associated with the position of $E_{\mathrm{F}}$ on the pseudogap. The DOS curves of the ordered intermetallic compound $\mathrm{Ti}_{3} \mathrm{Al}$ in the stable $\mathrm{DO}_{19}$ structure and the hypothetical $\mathrm{Ll}_{2}$ structure are shown in figure 1 . From figure 1 it can clearly be seen that the Fermi level falls exactly on the pseudogap for the stable $D_{19}$ structure and falls in the nonbonding region for the $\mathrm{Ll}_{2}$ structure. Maximum stability is ensured (Hong et al 1991; Xu and Freeman 1991; Xu et al 1991) if $W_{\text {occ }} / W_{\mathrm{b}} \approx 1$ where 


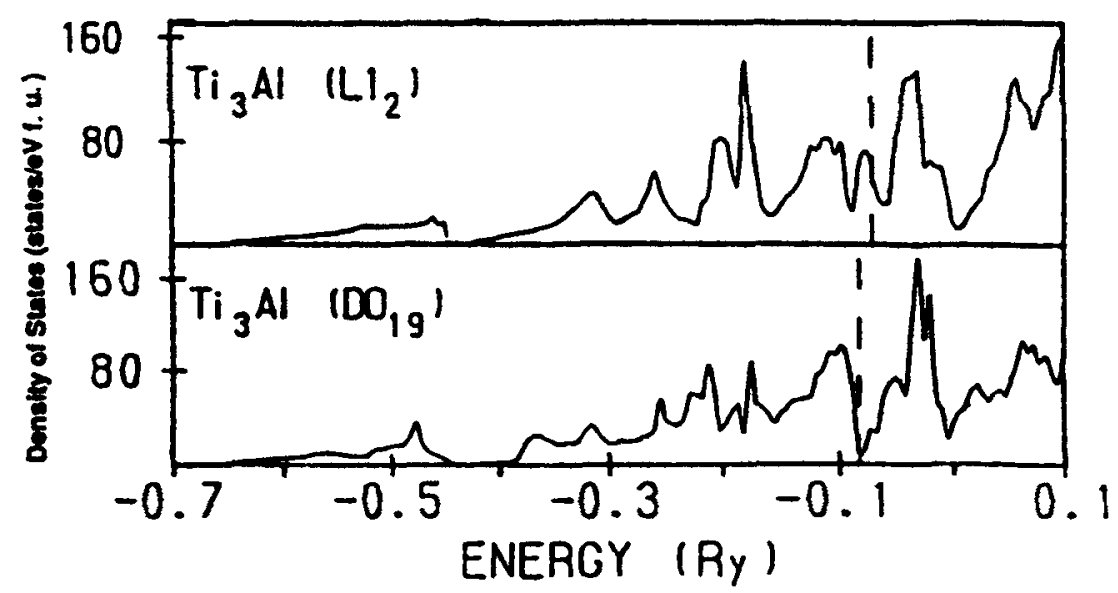

Figure 1. DOS curves of $\mathrm{Ti}_{3} \mathrm{Al}$ in stable $\mathrm{DO}_{19}$ and hypothetical $\mathrm{Li}_{2}$ structures.

$W_{\text {occ }}$ is the width of the occupied part of DOS and $W_{\mathrm{b}}$ the width of the region of the bonding states. This conclusion is further supported by the total energy curves (Hong et al 1991; Xu and Freeman 1991; Xu et al 1991). Hence, instead of looking for the total energy curves one can use the DOS histograms and the position of $E_{F}$ to find out the stable structure.

Apart from the study of structural stability, the density of states histogram provides a wealth of information. Firstly if the $E_{\mathrm{F}}$ lies exactly at the pseudogap, it refers to an ordered intermetallic compound of high melting point. If $E_{F}$ lies to the right of the pseudogap, i.e. the antibonding region, it refers to an unstable state and the system will exhibit tendency to be in the disordered or glassy state (Moruzzi et al 1983). Because the value of $N\left(E_{\mathrm{F}}\right)$ in this state is high, the system will exhibit fairly high superconducting transition temperature, high Pauli paramagnetic susceptibility and electronic specific heat coefficient (Ravindran and Asokamani 1995a). The high $T_{c}$ possessed by the system in the metastable phase is similar to the case of the A15 compounds and has a tendency to undergo structural transition, associated phonon softening and electronic topological transitions leading to shift in $E_{\mathrm{F}}$. Further, these systems with large $N\left(E_{\mathrm{F}}\right)$ will undergo martensitic transformation and hence often exhibit shape memory effects. Often the materials which have glass forming ability or martensitic transformation have these kind of features. If the $E_{F}$ falls in the higher energy side of the DOS curve or the antibonding states of the DOS curve, the system will be in the unstable state and try to go to some other configuration in order to minimize the energy. By alloying the elements with lower electron concentration in this type of compound one may succeed in stabilizing the metastable phase. On the other hand if the $E_{\mathrm{F}}$ lies to the left of the pseudogap, i.e. within the bonding states, not all bonding states are completely filled necessitating additional electrons to make the system more stable.

\section{Correlation between band filling of the bonding states and mechanical properties}

A correlation between the melting point and the density of states at the Fermi energy has been seen from the theoretical electronic structure calculations performed for the transition metal diborides (Wang et al 1994). A similar correlation has been 


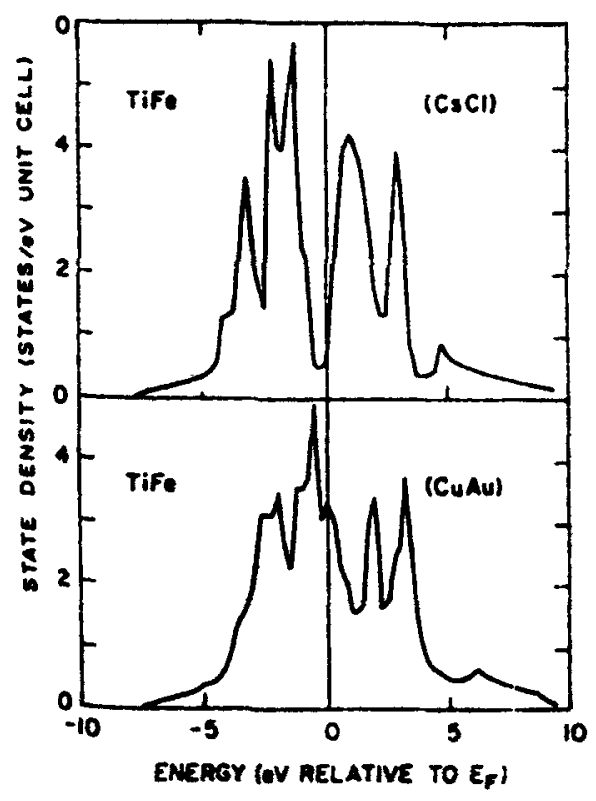

Figure 2. DOS curve of TiFe in bcc-like $\mathrm{CsCl}$ and fcc-like $\mathrm{CuAu}$ structures.

experimentally confirmed for a number of other alloys by Kuentzler and Waterstrat (1985, 1988). If the $E_{\mathrm{F}}$ falls on a peak in the DOS curve of the intermetallic compounds the self diffusion coefficient is seen to be enhanced resulting in a reduced creep resistance. This implies that the disordered alloys should be more ductile (Eberhart et al 1993). Further, even in the elastic regime Young's modulus $Y$ increases with increase of $d$ band filling as has been observed in $\mathrm{Ti}-\mathrm{Al}$ system (Collings et al 1970 ), where $E_{\mathrm{F}}$ falls on a pseudogap. The increase in the Young's modulus is due to the stiffening of the lattice.

A correlation has been established between electronic structure and glass-forming ability in transition metal compounds. The direct estimates of the coordination number by extended X-ray absorption fine structure show that the nearest-neighbours coordination found in glass is high. So, the $\mathrm{CsCl}$ type of TiFe is an example of ordered state and the $\mathrm{CuAu}$ type TiFe is equivalent to glassy state. In order to understand the role of electronic structure on glass-forming ability in transition metal compounds, bandstructure calculation has been performed (Moruzzi et al 1983) for TiFe in low coordination number ( $\mathrm{Fu} 1990), \mathrm{CsCl}$ type structure and the high coordination number (Sinha 1969; Liu 1984) CuAu type structure using the augmented spherical wave (ASW) method (Moruzzi et al 1983). The DOS curve of TiFe in both structures are shown in figure 2. In figure 2, near the centre of the DOS curve, this compound exhibits a deep valley in the bcc-like $\mathrm{CsCl}$ structure and a sharp peak in the fcc-like $\mathrm{CuAu}$ structure. The above evidence indicates rather large $N\left(E_{\mathrm{F}}\right)$ for those systems that form glasses, whereas systems that form ordered compound tend to have small $N\left(E_{\mathrm{F}}\right)$.

\section{Tailoring the properties of materials by tuning the Fermi level}

It is interesting to see that the $E_{\mathrm{F}}$ falls on the antibonding region in the DOS curve of metastable $\mathrm{ZrAl}_{3}$ (figure 3a), while in the case of $\mathrm{ScAl}_{3}$ it lies within the bonding region 

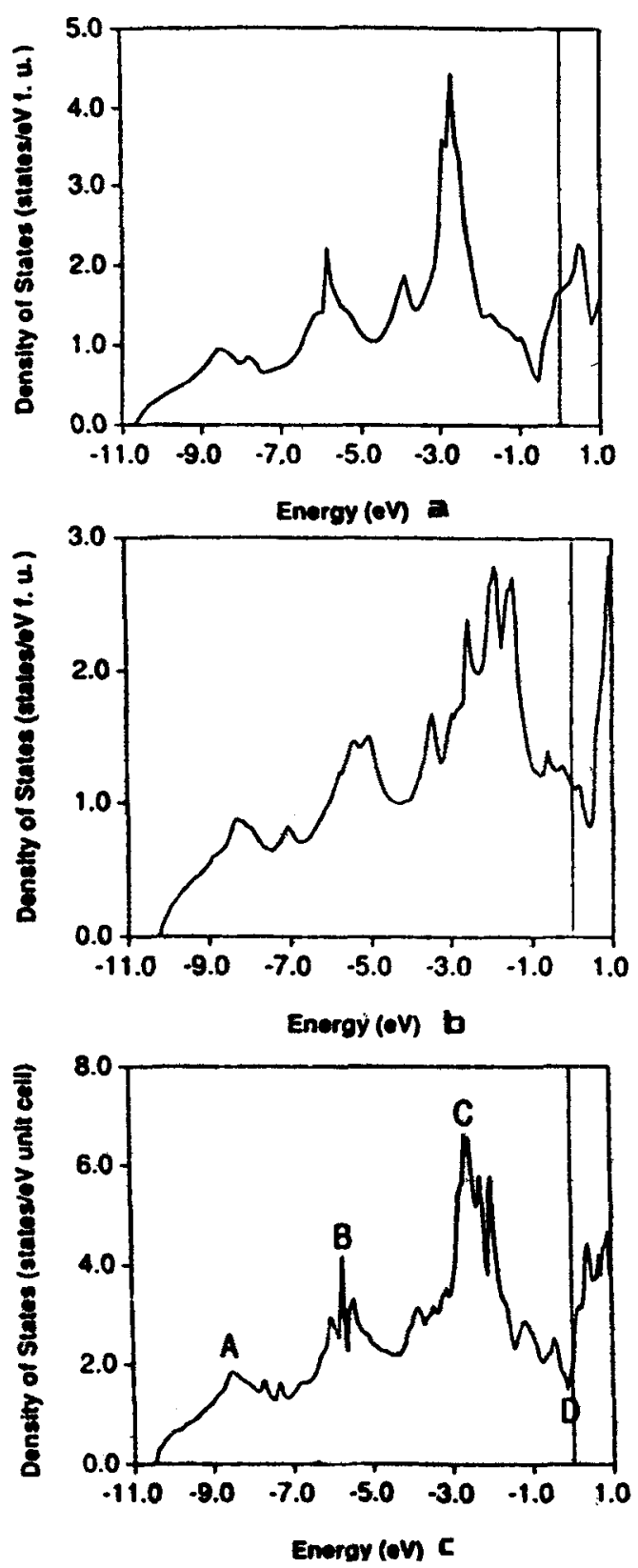

Figure 3. a. DOS curve of $\mathrm{ZrAl}_{3}$, b. DOS curve of $\mathrm{ScAl}_{3}$ and c. DOS curve of $\mathrm{Sc}_{0.5} \mathrm{Zr}_{0.5} \mathrm{Al}_{3}$.

(figure 3b) (Xu and Freeman 1990). Both $\mathrm{ScAl}_{3}$ and $\mathrm{ZrAl}_{3}$ are isostructural but $\mathrm{Sc}$ and $\mathrm{Zr}$ differ by valency one. One can now attempt to tune the Fermi level by mixing the tetravalent $\mathrm{Zr}$ and trivalent $\mathrm{Sc}$ atoms. Xu and Freeman (1990) showed that $W_{\text {ocd }} / W_{\mathrm{b}} \approx 1$ for $\mathrm{Sc}_{0.5} \mathrm{Zr}_{0.5} \mathrm{Al}_{3}$ (figure 3c) and this compound is found to be more stable than $\mathrm{ScAl}_{3}$ (figure $3 \mathrm{~b}$ ) and $\mathrm{ZrAl}_{3}$ (figure $3 \mathrm{a}$ ). This example demonstrates that the Fermi level can be tuned and a change in the valence electron concentration $e / a$ can be affected by suitable 

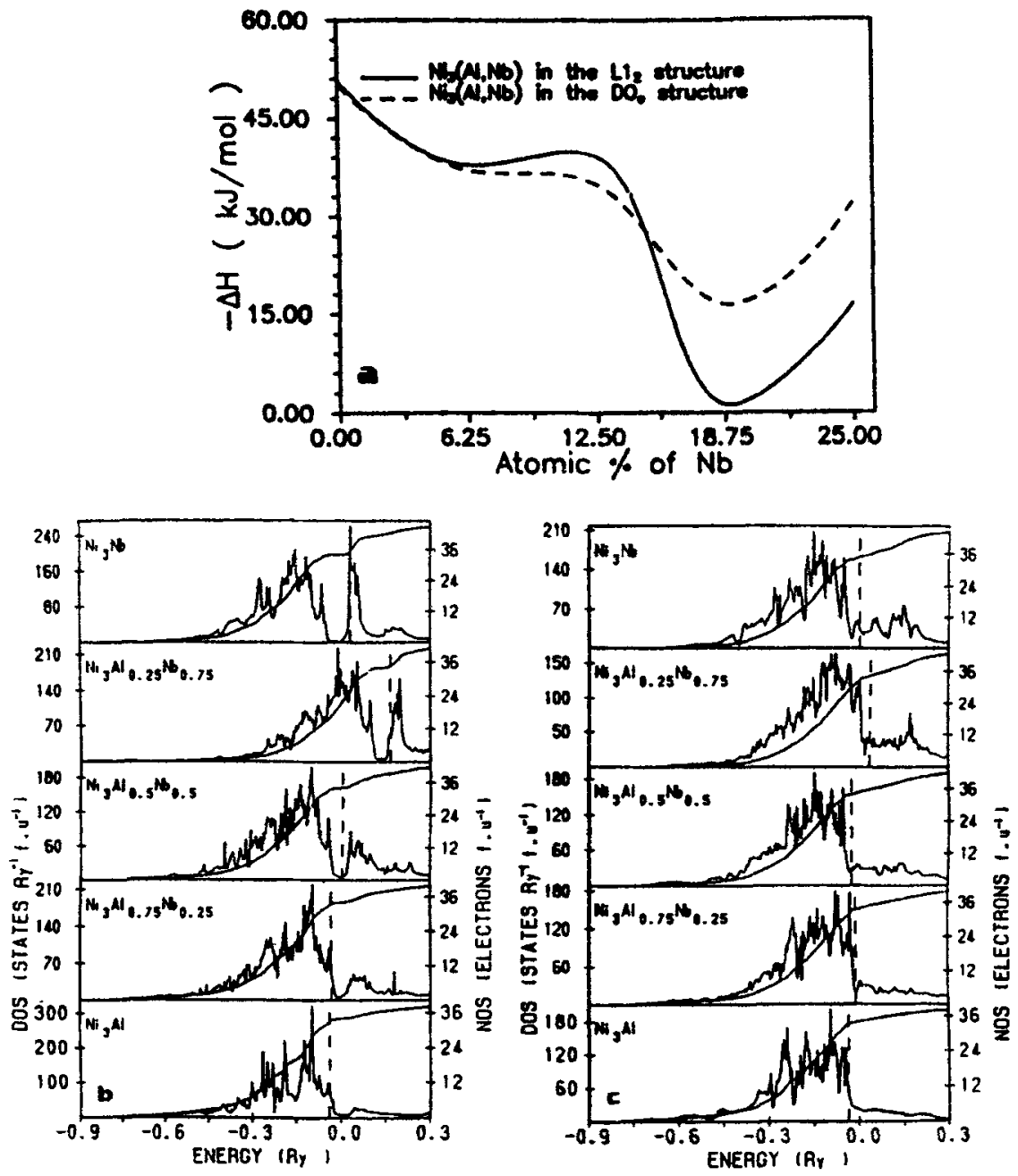

Figure 4. a. Heat of formation as a function of $\mathrm{Nb}$ substitution for $\mathrm{LI}_{2}$ and $\mathrm{DO}$, structures, b. DOS and NOS as a function of $\mathrm{Nb}$ substitution in $\mathrm{L1}_{2}$ structure and c. DOS and NOS as a function of $\mathrm{Nb}$ substitution in $\mathrm{DO}_{\mathrm{a}}$ structure.

alloying. This method of fixing the Fermi level at a determined position in the DOS curve to get the desired properties opens up ample scope for tailoring materials to attain desired properties. We now look into the correlation between $e / a$ ratio and structural stability.

The structural stability of alloys depend on $e / a$ values. For example, in the titaniumtransition metal compounds, if $e / a$ exceeds a value of about $4 \cdot 4$, the bcc $(\beta)$ structure remains stable against hcp $(\alpha)$ phase. In most of the $\mathrm{AB}$ type compounds, when $e / a \approx 5$, it stabilizes the $\mathrm{CsCl}$ structure consistent with the filling of maximum bonding states in the $\mathrm{CsCl}$ structure. We have made systematic electronic structure studies on $\mathrm{Ni}_{3} \mathrm{Al}$ as a function of $\mathrm{Nb}$ addition. The substitution of $\mathrm{Nb}$ in the $\mathrm{Al}$ site in $\mathrm{Ni}_{3} \mathrm{Al}$ increases the electron concentration of the system. At ambient condition $\mathrm{Ni}_{3} \mathrm{Al}$ is in the cubic $\mathrm{Ll}_{2}$ structure and $\mathrm{Ni}_{3} \mathrm{Nb}$ is in the orthorhombic $\mathrm{DO}_{\mathrm{a}}$ type structure. The heat of formation (figure $4 \mathrm{a}$ ) and DOS as a function of $\mathrm{Nb}$ substitution for $\mathrm{Ll}_{2}$ and $\mathrm{DO}_{\mathrm{a}}$ structures have 
been obtained using the tight binding linear muffin-tin orbital (TBLMTO) calculation and are given in figures $4 \mathrm{~b}$ and $\mathrm{c}$ respectively. From figure $4 \mathrm{~b}$ it is clear that the Fermi level is shifted from bonding states to antibonding state on increasing the electron concentration in the $\mathrm{Ll}_{2}$ structure. However in the $\mathrm{DO}_{\mathrm{a}}$ structure (figure $4 \mathrm{c}$ ), $\mathrm{Nb}$ addition retains $E_{\mathrm{F}}$ in the nonbonding state region of DOS. As the nonbonding state is favourable for stability as compared to a antibonding state the $\mathrm{Ll}_{2} \rightarrow \mathrm{DO}_{2}$ structural transition should take place as $e / a$ is increased by $\mathrm{Nb}$ addition. We do observe such a transition at $e / a=8.645$ (figure $4 a$ ) and this is found to be in good agreement with the values obtained from empirical correlation (Sinha 1969; Liu 1984). From figure 4a it is seen that the heat of formation of $\mathrm{Ni}_{3}(\mathrm{Al}, \mathrm{Nb})$ is greater than the weighted sum of that of $\mathrm{Ni}_{3} \mathrm{Al}$ and $\mathrm{Ni}_{3} \mathrm{Nb}$. This explains the experimental observation of multiphase formation in the $\mathrm{Ni}_{3}(\mathrm{Al}, \mathrm{Nb})$ system in the intermediate composition range.

\section{Correlation between the topology of the DOS curve and structural stability}

Earlier electronic structure studies on ordered intermetallic compounds have yielded a definite correlation between $\left\{N\left(E_{\mathrm{F}}\right)\right\}$ and their structural stability; low $N\left(E_{\mathrm{F}}\right)$ is associated with higher stability (Hong et al 1991; Xu and Freeman 1991; Xu et al 1991). The following analysis of the DOS curve gives information on structural stability of the materials. A material has high stability if (i) the conduction band is narrow, (ii) even if $E_{\mathrm{F}}$ is located on a peak of the DOS curve provided it is locally flat, (iii) $W_{\text {occ }} / W_{\mathrm{b}}$ is closer to 1 and (iv) conduction electron states accumulate in the relatively lower energy region. These criteria are as follows:

As mentioned by Hume-Rothery, among various possible crystal structures the one with the minimum conduction band width is most stable (Hume-Rothery 1963). From our detailed studies on various elements we have found that the above-said correlation exists for $s, p$ solids only. In general, the low conduction bandwidth indicates that the conduction electrons are in the lower energy region of the DOS curve. Hence it is expected that more stable state in the system is the one with the lower conduction bandwidth. However, in the case of transition metals where the $d$-electrons are mainly responsible for bonding and stability, this argument does not appear to be sound. On the other hand, the second criterion comes into force for such solids.

In order to understand this, consider a system with DOS curves in two different configurations as shown in figure $5 \mathrm{a}(1)$ and $5 \mathrm{a}(2)$. In the DOS curve in figure $5 \mathrm{a}(1)$ the $E_{\mathrm{F}}$ falls on a peak due to the presence of narrow band states. So, though the bandwidth in figure $5 \mathrm{a}(1)$ is lower than in the case of figure $5 \mathrm{a}(2)$ the position of $E_{\mathrm{F}}$ on the peak in figure 5a(1) implies small perturbation in the $E_{\mathrm{F}}$ to be associated with large changes in $N\left(E_{\mathrm{F}}\right)$. This leads to reduced stability of the structure of figure $5 \mathrm{a}(1)$. Further, most of the electrons in figure $5 \mathrm{a}(2)$ are accumulated in the lower energy region, indicating higher stability. If we consider two different configurations with DOS curves (figure $5 b(1)$ and figure $5 b(2)$ ), the stability of the system will be higher for figure $5 b(1)$ configuration than the other. Because, though the $N\left(E_{\mathrm{F}}\right)$ value and the conduction bandwidth of both configurations is the same, the $E_{\mathrm{F}}$ falls on a flat region of the peak in figure $5 b(1)$ and it falls in a sharp peak in figure $5 b(2)$. So, small shift in the $E_{F}$ will give large variation in the value of $N\left(E_{\mathrm{F}}\right)$ in figure $5 \mathrm{~b}(2)$ than figure $5 \mathrm{~b}(1)$. As a result of this the figure $5 \mathrm{~b}(2)$ configuration can be expected to be unstable. Our recent electronic structure studies on intermetallic compounds show that the often quoted correlation 

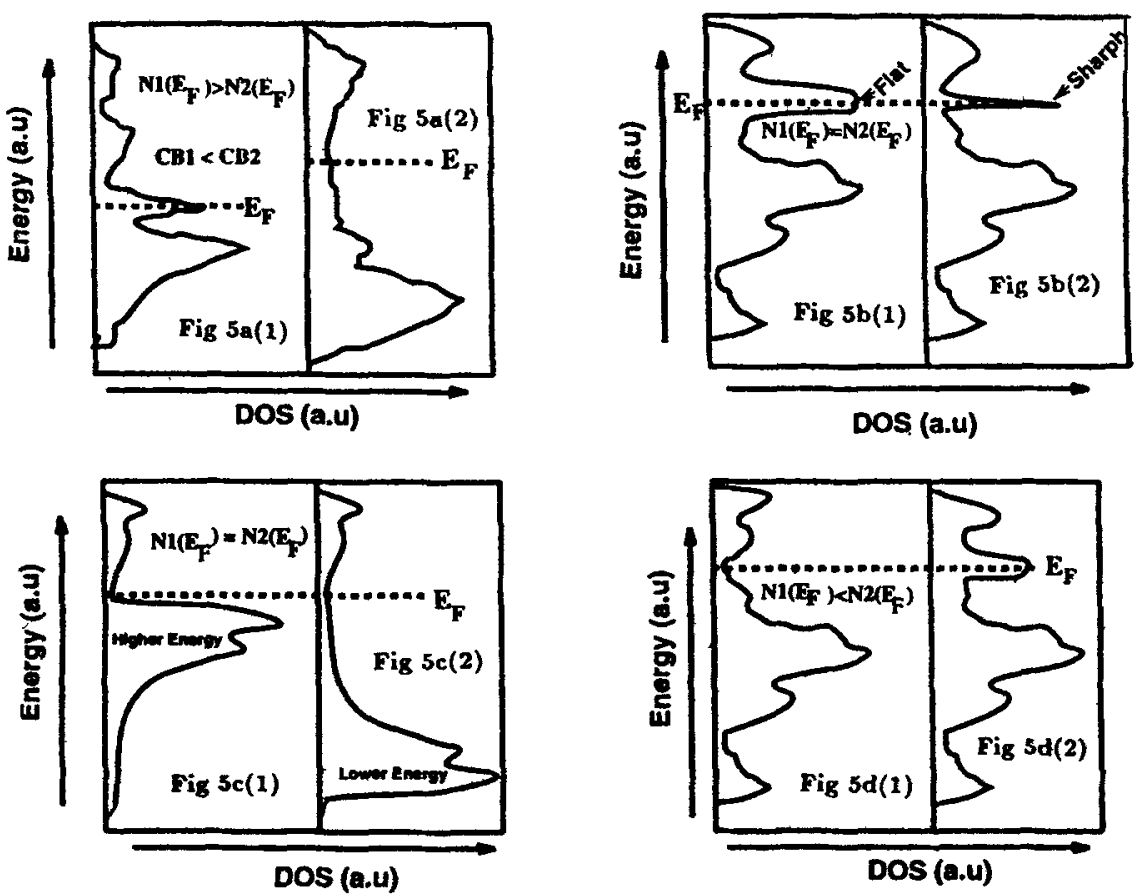

Figure 5. Position of $E_{\mathrm{F}}$ in the DOS curve and structural stability.

between stability with low $N\left(E_{\mathrm{F}}\right)$ is violated for materials which have metastability or martensitic transformation (Ravindran and Asokamani 1995b).

Let us consider a system which has the DOS curves in two different configurations as shown in figures $5 c(1)$ and $5 c(2)$. In both the cases, the conduction bandwidth is the same and also in both the DOS curves the $E_{\mathrm{F}}$ falls on a valley. But in figure $5 \mathrm{c}(2)$ most of the electrons are accumulated in the lower energy region providing the maximum weightage to electrons in the lower energy region. This ensures higher stability for the structure of figure $5 \mathrm{c}(2)$ as compared to that of figure $5 \mathrm{c}(1)$.

As mentioned earlier, there exists an inverse relation between structural stability and $N\left(E_{\mathrm{F}}\right)$ (Kuentzler and Waterstrat 1985, 1988; Fu 1990; Hong et al 1991; Xu and Freeman 1991; Zu et al 1991). If we consider a system with DOS curves figure $5 \mathrm{~d}(1)$ and figure $5 \mathrm{~d}(2)$ in two different configurations, the system will be more stable in figure $5 \mathrm{~d}(1)$ than in figure $5 \mathrm{~d}(2)$ according to the above correlation. This may be because in figure $5 \mathrm{~d}(1)$ a fewer number of electrons are found to accumulate in the vicinity of $E_{\mathrm{F}}$ as compared to the case of figure $5 \mathrm{~d}(1)$. Also in figure $5 \mathrm{~d}(1)$, small shifts in $E_{\mathrm{F}}$ does not have substantial influence on the value of $N\left(E_{\mathrm{F}}\right)$.

\section{Correlation between charge density distribution and ductility}

Single crystalline $\mathrm{ScAl}_{3}$ is found to be brittle, although its structure is cubic, ensuring the Von Mises criterion. This observation backed by recent investigations of transition metal aluminides leads to the conclusion that the crystallographic requirement of Von Mises has to be accompanied by certain electronic structure requirement for high 

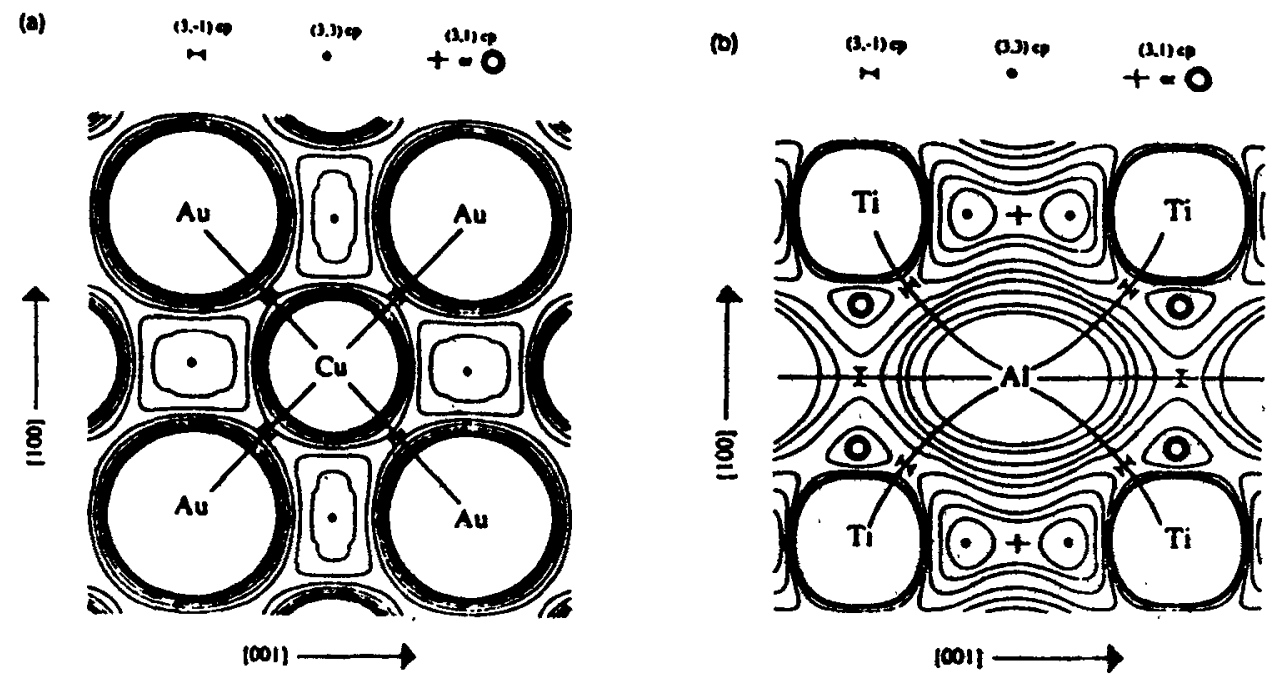

Figare 6. The charge density distribution of isostructural compounds $\mathrm{CuAu}$ (a) and $\mathrm{TiAl}(\mathrm{b})$

ductility. This view is further supported by the full potential linearized augmented plane wave calculations of $\mathrm{Fu}(1990)$. Fu clearly identified that the strong directional bonding is at the root of low ductility of the transition metal aluminides. The first principle studies of Eberhart et al (1993) clearly distinguished the ductile materials from the brittle materials using the correlation between charge density distribution and ductility. The charge density distribution of isostructural compounds $\mathrm{CuAu}$ and $\mathrm{TiAl}$ are given in figures $6 \mathrm{a}$ and $\mathrm{b}$ respectively. It is clearly seen that in the case of brittle TiAl (figure 6b) the charge density distribution is more anisotropic than in the ductile $\mathrm{CuAu}$ (figure 6a). The anisotropy in charge density distribution in $\mathrm{TiAl}$ is due to $\mathrm{Al}(\mathrm{p})-\mathrm{Ti}(\mathrm{d}) \sigma$-directional bonding. This directional bonding leads to high melting point and strength, but lower ductility. Pettifor (1970, 1992) analysed the structural maps and showed that there exists a possibility of moving from one structural domain to another by suitable addition of a third or more components. This provides a means of reducing directional bonding and thereby improves ductility.

\section{Conclusion}

From the falling of Fermi level in the DOS curve, we have qualitatively analysed the structural stability of intermetallics. Moreover various mechanical properties change with the variations in the topology of the DOS curve are discussed. These informations are useful to stabilize the metastable phases and also to obtain tailormade property materials by proper alloying. Because of the filling of antibonding states by increase of $e / a$ values, the systems prefer to go from one structure to the other. Though the total energies obtained from the first principle band structure calculation quantitatively predict the stability of the materials, from the analysis of filling of bonding, nonbonding and antibonding states one can tailor the properties of materials by tuning the Fermi level. 


\section{Acknowledgements}

One of the authors (PR) thanks Dr H K Sahu for discussions and critical reading of the manuscript and Dr T S Radhakrishnan for his encouragement. The authors wish to thank CSIR, New Delhi for the financial support.

\section{References}

Andersen O K 1975 Phys. Rev. B12 3060

Briggs C W (ed.) 1992 The science of new materials (Oxford: Blackwell) p. 86

Collings E W, Enderby J E, Gegel H L and Ho J C 1970 in The science and technology and application of titanium, Proc. of an int. conf. (eds) R I Jaffee and N E Promisel (New York: Plenum Press) Vol. 2, p. 801

Eberhart M E, Clougherty D P and MacLaren J M 1993 J. Mater. Res. 8438

Fu C L 1990 J. Mater. Res. 5971

Gelatt C D, Williams A R Jr. and Moruzzi V L 1983 Phys. Rev. B27 2005

Hong T, Yang T J W, Guo X Q, Freeman A J, Oguchi T and Xu J H 1991 Phys. Rev. B43 1940

Hume-Rothery W 1963 Electrons, atoms, metals and alloys (New York: Dover Publications)

Kuentzler R and Waterstrat R M 1985 Solid State Commun. 54517

Kuentzler R and Waterstrat R M 1988 Solid State Commun. 6885

Liu C T 1984 Int. Met. Rev. 29168

Von Mises R 1928 Z. Angew. Math. Mech. 8161

Moruzzi V L, Janak J F and Williams A R 1978 Calculated electronic properties of metals (New York: Pergamon Press Inc.)

Moruzzi V L, Oelhafen P and Williams A R 1983 Phys. Rev. B27 7194

Pastural A, Colinet C and Hicter P 1985 Physica B132 177

Pettifor D G 1970 J. Phys. C3 367

Ravindran P and Asokamani R 1995a J. Phys.: Condens. Matter 75567

Ravindran P and Asokamani R 1995b Phys. Rev. BSO 668

Sinha A K 1969 Trans. Am. Inst. Mech. Eng. 245911

Skriver H L 1985 Phys. Rev. B31 1909

Wang X B, Tian D C and Wang L 1994 J. Phys.: Condens. Matter 610185

Xu J H and Freeman A J 1990 Phys. Rev. B41 12553

Xu J H and Freeman A J 1991 J. Mater. Res. 61188

Xu J H, Oguchi T and Freeman A J 1991 Phys. Rev. B36 4186 